\title{
Anabases
}

ANABASES Traditions et réceptions de l'Antiquité

1 | 2005

Varia

\section{Grecs, Romains et Germains au XIXe siècle : quelle Antiquité pour l'État national allemand?}

Hinnerk Bruhns

\section{(2) OpenEdition}

1 Journals

Édition électronique

URL : http://journals.openedition.org/anabases/1257

DOI : 10.4000/anabases. 1257

ISSN : 2256-9421

Éditeur

E.R.A.S.M.E.

Édition imprimée

Date de publication : 1 mars 2005

Pagination : 17-43

ISSN : 1774-4296

\section{Référence électronique}

Hinnerk Bruhns, «Grecs, Romains et Germains au XIXe siècle : quelle Antiquité pour l'État national allemand? ", Anabases [En ligne], 1 | 2005, mis en ligne le 01 septembre 2011, consulté le 20 octobre 2019. URL : http://journals.openedition.org/anabases/1257 ; DOI : 10.4000/anabases. 1257

(c) Anabases 
Anabases 1 (2005), p. 17-43

\section{Grecs, Romains et Germains au XIXe siècle: quelle Antiquité pour l'État national allemand ?}

HINNERK BRUHNS

\section{Du bon ou du mauvais usage de l'Antiquité}

LE 27 JANVIER 1897, Ulrich von Wilamowitz-Moellendorff, illustre helléniste et gendre de Theodor Mommsen, prononça, au nom de l'Université de Göttingen, un "Discours en l'honneur de l'anniversaire de sa Majesté, notre Empereur et Roi», Guillaume II. Wilamowitz aborda, dès les premières phrases de ce discours solennel, intitulé Weltperioden (Périodes de l'histoire mondiale), la question de l'unité des Staaten und Stämme, des États et peuples ${ }^{1}$ allemands, unité qui venait d'être renforcée par un nouveau lien, le Code civil allemand (Deutsches bürgerliches Gesetzbuch). L'orateur rappela ensuite que l'Allemagne se préparait à commémorer le centenaire de la naissance, en 1797, du premier empereur allemand, Guillaume I ${ }^{\mathrm{er}}$, couronné en $1871^{2}$. L'évocation de ce centenaire était pour Wilamowitz un prétexte pour s'interroger sur le sens de l'histoire allemande entre ces deux dates, 1797 et 1897. Car le hasard avait voulu que le premier empereur allemand naquît dans l'année même qui symbolisait pour les Allemands la mort du Heiliges Römisches Reich deutscher Nation - même si, formellement, le certificat de décès du Reich ne fut établi

$1 \quad$ Wilamowitz emploie le terme de Stamm (tribu, peuplade), qui a remplacé au cours du XIX ${ }^{e}$ siècle, sous l'impulsion des médiévistes, le terme de Volk appliqué auparavant aux peuples allemands (Francs, Saxons, Alamans, etc.).

2 Né en 1797, couronné roi de Prusse en 1861, empereur allemand en 1871 et décédé en 1888. 
qu'en $1806^{3}$. En 1797, en effet, l'empereur François II avait cédé à la France, par le traité de Campo-Formio, les provinces belges de l'Autriche et lui avait surtout abandonné la possession de la rive gauche du Rhin et de Mayence. En cette même année 1797, Frédéric Guillaume III, père du futur empereur, accédait au trône de la Prusse, un État dont Wilamowitz parle en ces termes :

[ein Staat] «der kaum deutsch genannt zu werden verdiente, der vielmehr erst zertrümmert werden mußte, damit jenes Preußen entstünde, dessen Volk sich durch eignen Opfermut die Anwartschaft auf die Führung Deutschlands erwerben sollte.» 4

Un siècle après ces événements, Wilamowitz hésite quant à savoir si le bilan pour le peuple allemand se présentait plus favorablement à la fin du XIXe ou à la fin du XVIII ${ }^{\mathrm{e}}$ siècle, à une époque où des " hommes allemands " avaient érigé, pendant les dix années de paix obtenues grâce au traité " honteux ", au bénéfice de l'humanité toute entière, les Ideale höchster Menschenbildung, à présent menacés partout par le «stupide fanatisme national»; et l'orateur de se demander si vraiment, un jour lointain, les temps si opposés de Goethe et de Bismarck pourront être considérés comme deux moments d'une seule et même époque.

Vingt ans auparavant, en 1877, Wilamowitz avait donné à son premier discours en l'honneur d'un anniversaire impérial le titre panégyrique de «Von des attischen Reiches Herrlichkeit». Dans ce texte, l'empire allemand apparaissait plus magnifique que l'Athènes de Périclès, et l'orateur n'y évoquait ni les dangers qui menacent «unser junges Reich, [...] unsere alte Kultur» 5, ni le moindre doute quant à la compatibilité de l'État national moderne avec les valeurs de la culture classique. Vingt ans plus tard, en 1897, l'enthousiasme de la jeunesse - Wilamowitz avait prononcé ce premier discours à l'âge de vingt-neuf ans - et l'adhésion enthousiaste à l'État national et à l'unité allemande avaient cédé le pas à un scepticisme quelque peu désabusé. Ce sentiment n'était pas réservé aux seuls représentants de la culture classique, même si

3 On connaît le sarcastique éloge funèbre prononcé par le jeune Görres: «Am 30. Dezember 1797, am Tage des Übergangs zu Mainz nachmittags um 3 Uhr starb zu Regensburg in dem blühenden Alter von 955 Jahren 5 Monaten und 28 Tagen sanft und selig an einer gänzlichen Entkräftung und hinzugekommenem Schlagfuß bei völligem Bewußtsein und mit allen heiligen Sakramenten versehen das heilige römische Reich schwerfälligen Angedenkens.», soit "Le 30 décembre 1797, le jour de la reddition de Mayence, à trois heures de l'après-midi, à l'âge florissant de 955 ans, 5 mois et 28 jours, s'est éteint à Ratisbonne, dans la paix du Seigneur, victime d'un épuisement total et frappé en outre d'apoplexie, en pleine conscience et pourvu de tous les saints sacrements, le Saint Empire Romain de lourde mémoire», cité par K.O. Freiherr v. ARETIN, Vom Deutschen Reich zum Deutschen Bund, Göttingen, 1980, p. 84.

4 «... un État qui mériterait à peine d'être appelé allemand, qui au contraire a dû d'abord être démoli pour que naisse cette Prusse dont le peuple devait, en se sacrifiant avec courage, acquérir le droit d'exercer la direction de l'Allemagne.» U. v. WILAMOWITZMOELLENDORFF, "Weltperioden. Rede zu Kaisersgeburtstag 1897", in Reden und Vorträge, Berlin, 1901, p. 120-135, en part. p. 121.

5 "Notre jeune empire [...] notre vieille civilisation", discours de 1897, in Reden und Vorträge, p. 82. 
ceux-ci avaient des raisons particulières de ressentir un malaise face à l'évolution intérieure du Reich dont la modernisation s'accompagnait d'une critique du trop grand poids accordé à l'Antiquité dans la formation des élites allemandes.

L'Allemagne de Goethe, ou bien celle de Bismarck et de Guillaume II: face à une telle alternative, la question de savoir si l'État-Nation ou «les partisans de l'idée nationale ont utilisé l'Antiquité (classique) pour soutenir l'idée de Nation» ${ }^{6}$ peut, à première vue, paraître relativement éloignée non seulement des enjeux idéologiques qui traversaient le nouvel État, mais aussi de ceux de la grande époque du mouvement national allemand jusqu'à l'échec de la révolution de 1848 .

En effet, parmi les nombreuses études récentes consacrées à la construction de la nation, des identités ou des imaginaires nationaux en Allemagne, très rares sont celles qui posent la question de l'utilisation de l'Antiquité. Un titre comme Die Antike als Begründung des deutschen Nationalbewußtseins (L'Antiquité comme fondement de la conscience nationale allemande), titre d'un article publié en 1985 mais dont l'idée et la première version remontent en fait à l'année 1942, semblerait tout à fait improbable dans les travaux des historiens contemporanéistes et des sociologues qui s'intéressent aujourd'hui à la question de la légitimation des nations et des nationalismes par le recours à l'Histoire. Il est symptomatique que les Deutsche Erinnerungsorte 7 , les Lieux de mémoire allemands, conçus sur le modèle de l'entreprise de Pierre Nora, ne répertorient aucun lieu de l'Antiquité classique: ni la Troie de Schliemann, ni les fouilles d'Olympie ou de Pergame, ni même la si populaire collection Die schönsten Sagen des klassischen Altertums de Gustav Schwab qui, pendant un siècle et demi, a formé l'image qu'avaient la jeunesse allemande et plus largement les classes cultivées, de la Grèce antique et de la fondation de Rome ${ }^{8}$. Certes, on n'a pas oublié que le siècle du mouvement national et de l'unité allemande a été la grande époque des études classiques et le siècle de l'histoire, ni que de grands historiens de l'Antiquité, en premier lieu Droysen et Mommsen, ont occupé une place exceptionnelle à la fois dans l'histoire de leur discipline et dans celle du mouvement national allemand. L'absence, au moins relative, de l'Antiquité dans les études récentes sur la "construction" de la nation allemande correspond, assurément, à la disparition progressive de l'Antiquité de l'horizon intellectuel et culturel des spécialistes des questions nationales modernes. Mais ce n'est pas la seule raison: une autre se trouve, à mon avis, dans la nature ambiguë et complexe du rapport entre l'idée nationale et l'Antiquité dans l'Allemagne du XIXe siècle.

6 Question de recherche posée dans l'appel à communications pour la journée d'études du groupe E.R.A.S.M.E. à Toulouse, le 2 avril 2004.

$7 \quad$ E. FRANÇOIS \& H. SCHULZE (éd.), Deutsche Erinnerungsorte, 3 vol., Munich, 2001.

8 Première publication, en trois volumes: 1838-1840. L'Antiquité apparaît dans les Deutsche Erinnerungsorte à travers la figure d'Arminius et le roman de Felix Dahn, Ein Kampf um Rom. Cf. infra, p. 38-40 sv. 
Pour répondre à la question posée par les organisateurs de la journée d'études, on pourrait s'intéresser d'entrée de jeu à l'impact, sur le mouvement national allemand, de l'image d'Arminius, haud dubie liberator Germaniae 9, vainqueur des Romains et de Napoléon, ou de celle d'Alexandre le Grand, roi des Macédoniens et des Prussiens, unificateur de la Grèce et de l'Allemagne, ou encore de celle de César qui, une fois pour toutes, avait démontré, devant l'Histoire Universelle, comment parachever l'unification d'une nation. De telles analogies historiques et leurs utilisations politiques (nous y reviendrons) pourraient créer l'illusion d'un rapport simple et positif, en Allemagne, entre Nation et Antiquité. Je partirai d'une affirmation plus nuancée: l'utilisation de modèles et références antiques, autant à l'intérieur du mouvement national allemand que, plus tard, dans les cercles officiels du nouvel État national, font apparaître un rapport plus difficile et polymorphe que ne le suggèrent les analogies précitées. La relation à l'Antiquité est ambiguë: elle se fonde sur des idées contradictoires, se définit, nécessairement, par rapport aux situations politiques intérieures et extérieures du moment et se transforme en fonction des évolutions à la fois de l'histoire allemande et de l'Altertumskunde, la science de l'Antiquité.

Un épisode de l'année 1890, deux décennies après la création de l'État national allemand, pourra illustrer un aspect de cette relation ambiguë. La réforme du système éducatif était considérée comme une nécessité impérative pour faire de l'Allemagne une nation moderne, de la même façon qu'une réforme analogue avait été un enjeu central du mouvement national au début du siècle. Une des toutes premières initiatives prises par l'empereur Guillaume II en 1890, après avoir renvoyé Bismarck, visait la réforme des lycées. Lors d'une Schulkonferenz, conférence sur l'éducation scolaire, préparée à la hâte, l'Empereur intervint, avec un discours improvisé, pour encourager le renforcement des Realschulen au détriment des lycées classiques. Â ces derniers, il reprochait un manque de fondement national: «[...] da fehlt es vor allem an der nationalen Basis. Wir müssen als Grundlage für das Gymnasium das Deutsche nehmen; wir sollen nationale junge Deutsche erziehen und nicht junge Griechen und Römer.» ${ }^{10} \mathrm{Au}$ centre de l'éducation dans les lycées devrait être placée, selon

9 Tacite, Annales II, 88. R. WIEgELS \& W. WOESLER (éd.), Arminius und die Varusschlacht. Geschichte-Mythos-Literatur, Paderborn, 1995. W.M. DOYE, "Arminius", in Deutsche Erinnerungsorte, 3, p. 567-602 ; Ch. TACKE, Denkmal im sozialen Raum. Nationale Symbole in Deutschland und Frankreich im 19. Jahrhundert, Göttingen, 1995.

10 «Ce qui manque surtout, c’est le fondement national. Les programmes du lycée doivent reposer sur l'enseignement de l'allemand; nous devons éduquer de jeunes Allemands nationaux, et non pas de jeunes Grecs et Romains.» Cf. M. LANDFESTER, “Griechen und Deutsche. Der Mythos einer Wahlverwandtschaft", in H. BERDING (éd.), Mythos und Nation. Studien zur Entwicklung des kollektiven Bewußtseins in der Neuzeit, vol. 3, Francfort, 1996, p. 215. Cf. aussi le discours pour la défense des langues anciennes et de la culture classique (1892, Université de Göttingen), cf. WILAMOWITZ, "Philologie und Schulreform", in Reden und Vorträge, p. 97-119. L.C ANFORA, "Wilamowitz und die Schulreform: das "Griechische Lesebuch»", in W.M. CALDER III, H. FLASHAR \& Th. LINDKEN (éd.), Wilamowitz nach 50 Jahren, Darmstadt, 1985, p. 632-648. 
Guillaume II, «der deutsche Aufsatz», la dissertation allemande. On pourrait croire que le jeune empereur voulait surtout favoriser une modernisation de la formation des élites allemandes, modernisation indispensable dans un État qui s'industrialisait à toute vitesse. Ce motif était important. Mais tout aussi importante était la nécessité de faire progresser, une fois achevée la äussere Staatsbildung, la formation extérieure de l'État, l'objectif de la innere Staatsbildung, de la formation intérieure de l'État, qui était loin d'être accomplie. L'Allemagne de Bismarck était traversée par deux grands conflits, d'une part avec l'Église catholique (le Kulturkampf, 1871-1887), d'autre part avec les socialistes (loi contre les socialistes, 1878). L'échec de la révolution de 1848 avait divisé la bourgeoisie et le mouvement unitaire qui ne s'étaient pas entièrement ralliés à Bismarck et à sa politique, dans la mesure où elle avait fait déboucher le rêve du mouvement national sur un Deutsches Reich obtenu par la guerre et proclamé à l'étranger, au château de Versailles, en 1871.

Qu'il y eût des contradictions entre les contenus de l'éducation classique et la formation «de jeunes Allemands nationaux», comme le constatait Guillaume II, ce n'était pas une nouveauté. On verra plus loin que, depuis la moitié du siècle, les milieux éducatifs s'étaient efforcés, non sans difficultés, de réconcilier valeurs nationales et valeurs de l'Antiquité grecque et romaine. L'attaque frontale contre l'enseignement du grec et du latin, et la proposition de fonder l'éducation sur la dissertation allemande, traduisaient donc un sentiment de malaise répandu. Que la place de l'Antiquité dans l'éducation et la culture allemandes posât un grave problème, ce n'était d'ailleurs ni seulement une idée impériale, ni uniquement une préoccupation du moment. Une génération plus tard, après la Première guerre mondiale, le culte absolu voué par les Allemands à la pensée et à la culture grecques était considéré comme une des racines de l'isolement non seulement politique, mais aussi intellectuel de l'Allemagne ${ }^{11}$. Nation et Antiquité formaient donc apparemment, en Allemagne, un couple ambigu, aux relations complexes et contradictoires. Pour éclairer cette relation, nous devons remonter d'abord à la fin du XVIII $^{\mathrm{e}}$ et au début du XIX ${ }^{\mathrm{e}}$ siècle, avant de revenir sur le moment de la fondation de l'État unitaire allemand en 1871.

\section{La Grèce des Allemands : du mythe grec à l'œuvre nationale des fouilles d'Olympie}

Jusqu'à la fin du XVIII siècle, les Allemands partageaient avec la France et d'autres pays européens une même culture classique, fondée largement sur l'enseignement de

11 C'est ce que constate Ludwig Curtius en 1926, citant l'opinion de savants étrangers; cf. W. RÜEGG, "Die Antike als Begründung des deutschen Nationalbewußtseins", in W.S CHUller (éd.), Antike in der Moderne, Constance, 1985, p. 267-287, citation p.268. 
Cicéron et de la rhétorique. La modification radicale, en Allemagne, du rapport des élites à cette tradition est un des éléments clés de la différence entre la France et l'Allemagne au XIXe siècle. Ce changement fut, en grande partie, une réaction à la Révolution française et à Napoléon. En simplifiant, on pourrait dire que la dévalorisation des références romaines en Allemagne correspondait à leur revalorisation en France. Puisque la France s'identifiait, à cette époque, de plus en plus à Rome, celle-ci ne pouvait être l'idéal ou la référence antique principale du mouvement unitaire allemand ${ }^{12}$.

L'utilisation politique et idéologique anti-française d'Arminius ${ }^{13}$ au XIX ${ }^{\mathrm{e}}$ siècle faisait écho à l'identification de la France avec Rome. Si la défaite de Vercingétorix avait "permis en réalité à la France d'accepter Rome, de se faire l'héritière de Rome» ${ }^{14}$, la victoire des "Germains» sur Rome, à laquelle s'ajoutait le mythe de la "germanité», fabriqué par les humanistes à partir de la Germania de Tacite, traçait une voie dans la direction inverse. À l'époque des guerres de libération et jusqu'après la guerre de 1870, le souvenir de la résistance victorieuse des Germains face à Rome fut instrumentalisé au service de la lutte des Allemands contre Napoléon et la France ${ }^{15}$. Et par rapport à la situation intérieure des royaumes et principautés allemandes rassemblés dans le Deutscher Bund, ni la Rome républicaine, antimonarchique, ni la Rome des Césars ne pouvaient fournir de modèle politique ou servir de réservoir rhétorique pour le mouvement national allemand, même si l'Allemagne connut également un mythe napoléonien ${ }^{16}$.

Restait donc la Grèce. Avec l'inconvénient, pourtant, qu'il était difficile de présenter les Grecs comme les champions de l'idée et de la réalisation d'un État national. L'identification à la Grèce antique fonctionnait même plutôt dans le sens contraire, c'est-à-dire dans le sens d'une valorisation de la Kulturnation au détriment de l'idée de la Staatsnation. On trouve encore un écho à cette conception très

12 Pour la problématique de l'interaction dans les relations franco-allemandes, cf. M.J EISMANN, Das Vaterland der Feinde. Studien zum nationalen Feindbegriff und Selbstverständnis in Deutschland und Frankreich 1792-1918, Stuttgart, 1992 (trad. fr.: La Patrie de l'ennemi. La notion d'ennemi national et la représentation de la nation en Allemagne et en France de 1792 à 1918, Paris, 1997).

13 Dès le XVI siècle, le nom d'Arminius a été considéré, par erreur, comme l'équivalent du nom allemand Hermann; par l'ajout du nom de la peuplade des Cherusci, Arminius est devenu pour les Allemands: Hermann der Cherusker.

14 C. NICOLET, La fabrique d'une nation. La France entre Rome et les Germains, Paris, 2003, p. 277.

15 Au XVII e siècle, c'est l'empereur Léopold Irr qui s'était servi de l'identification avec Arminius pour souligner l'importance de ses victoires sur les Turcs. La contradiction avec l'idée de la translatio imperii, élément important de l'idéologie impériale des Habsbourg, ne gênait apparemment que peu. Cf. DOYE, "Arminius", p. 592.

16 W. WÜLFING, "Heiland" und "Höllensohn». Zum Napoleon-Mythos im Deutschland des 19. Jahrhunderts", in BERDING (éd.), Mythos und Nation, vol. 3, p.164-184; cf. aussi H. SCHULZE, "Napoleon", in FRANÇOIS \& SCHULZE, Erinnerungsorte, vol. 2, p. 28-46 et 686-687. 
répandue dans un important petit livre sur l'histoire de la conscience nationale allemande, écrit au milieu de la Première Guerre mondiale. L'auteur, Paul Joachimsen, y constate que, dans l'histoire grecque, «l'État national n'apparaît que comme un fantôme dans les constructions mentales de Gorgias et d'Isocrate». À l'instar des Grecs, les Allemands semblaient donc devoir en rester au stade de la Kulturnation, d'une nation qui n'existait que par une culture commune. L'analogie fonctionnait d'ailleurs dans les deux sens: déjà Barthold Georg Niebuhr (1776-1831) avait appelé la Grèce «l'Allemagne de l'Antiquité» 17. Présenter l'Allemagne comme la Grèce de l'époque moderne pouvait en réalité faire partie d'une stratégie de mise en valeur de la situation particulière de l'Allemagne, même au plan politique. C'était aussi une tentative d'accorder une valeur supérieure au concept et à la réalité de Kulturnation par rapport au nouveau concept de nation politique, issu de la Révolution française. Néanmoins, ce sont la Grèce et l'histoire grecque qui devaient jouer, au cours du XIXe siècle, un rôle important non seulement dans le "fondement de la conscience nationale allemande" ${ }^{18}$, mais également comme catalyseur dans la relation tendue entre les idées de Nation, État et Antiquité.

De l'évolution de cette relation particulière de l'Allemagne à la Grèce, à travers le $\mathrm{XIX}^{\mathrm{e}}$ siècle, nous ne retiendrons ici que quelques étapes significatives ${ }^{19}$ qui marquent l'éloignement de l'Allemagne, à partir de la fin du XVIIIe siècle, d'une tradition européenne commune, fondée et représentée par Winckelmann et ses successeurs (en Allemagne notamment Schiller et Goethe), qui avaient découvert dans l'Antiquité grecque «un lien de consubstantialité entre la beauté (naturelle et artistique), le bonheur (individuel et collectif) et la liberté (du citoyen et de la cité)...» ${ }^{20}$.

À partir des années 1770, en réaction d'abord contre l'État absolutiste, ensuite contre la Révolution française qui cherchait ses références dans l'Antiquité romaine, un «mythe grec allemand» (der deutsche Griechenmythos) se superposa au mythe grec européen et se transformait en un mythe allemand, national, de la supériorité culturelle de l'Allemagne (Schlegel, Humboldt, Herder). La parenté avec la nation élue des Grecs anciens élevait les Allemands eux-mêmes au rang d'une nation élue, nation au sens de Kulturnation. Dans ce contexte, la multiplicité des États et structures étatiques en Allemagne n'était pas considérée comme un phénomène négatif mais, au contraire, comme la condition de la supériorité nationale au plan culturel. Cette première étape est celle du Neuhumanismus qui affirme l'identité

17 P. JOACHIMSEN, Vom deutschen Volk zum deutschen Staat. Zur Geschichte des deutschen Nationalbewußtseins, $2^{\mathrm{e}}$ éd. modifiée, Leipzig et Berlin, 1920 (1 ${ }^{\mathrm{e}}$ éd., 1916), citation p.6.

18 Cf. RÜEGG, "Die Antike als Begründung”, p. 267-287.

19 M. LANDFESTER, "Griechen und Deutsche: Der Mythos einer "Wahlverwandtschaft»", in B ERDING (éd.), Mythos und Nation, vol. 3, p. 198-219.

20 L. CALVIÉ, "Antiquité et Révolution françaises dans la pensée et les lettres allemandes à la fin du XVIII e siècle", Annales historiques de la Révolution française, 317/3 (1999), p.455-475, citation p. 473 . 
profonde entre Allemands et Grecs anciens. Pour Wilhelm von Humboldt ${ }^{21}$, qui par ses réformes de l'enseignement secondaire et supérieur en Prusse (1809-1810) et par la fondation de l'Université de Berlin, en 1810, a marqué durablement la vie culturelle allemande, cette identification à la Grèce classique n'avait pas, dans un premier temps, pour finalité la nation et la conscience nationale, mais l'idée que la culture grecque et la culture allemande, plus précisément la deutsche Klassik, étaient l'incarnation (Verkörperung) des potentialités optimales de l'humanité. Les conséquences de cette conception sur la perception et sur le traitement de l'histoire grecque antique ont été exprimées par W. v. Humboldt en ces termes:

«Es ist daher mit dem Studium der Griechischen Geschichte für uns nicht, wie mit dem der Geschichte anderer Völker. Die Griechen treten gänzlich aus dem Kreise derselben heraus [....] und wir verkennen durchaus unser Verhältnis zu ihnen, wenn wir den Massstab der übrigen Weltgeschichte auf sie anzuwenden wagen. Ihre Kenntniss ist uns nicht bloss angenehm, nützlich und nothwendig, nur in ihr finden wir das Ideal dessen, was wir selbst seyn und hervorbringen möchten; wenn jeder andre Theil der Geschichte uns mit menschlicher Klugheit und menschlicher Erfahrung bereichert, so schöpfen wir aus der Betrachtung der Griechen etwas mehr als Irrdisches, ja beinah Göttliches.» 22

Le nouveau mythe grec allemand était le produit artificiel d'une élite intellectuelle et fut contesté dès la génération suivante, celle des Romantiques, qui cherchait les racines de la véritable Allemagne dans une vision idéalisée du Moyen Âge ${ }^{23}$. Pourtant, dans les institutions d'éducation, lycées et universités, son succès fut quasi total et il devint, pendant un siècle, un élément constitutif de la culture et de la conscience de soi des élites cultivées, en particulier du Bildungsbürgertum qui le perpétuait.

21 Pour une analyse détaillée et les différences par rapport à la vision de la Grèce chez Schiller d'une part, chez Hegel d'autre part, voir J. QUILLIEN, G. de Humboldt et la Grèce, Lille, 1983. Cf. également M. KRAUL, "Bildung und Bürgerlichkeit", in J.K OCKA (éd.), Bürgertum im 19. Jahrhundert. Deutschland im europäischen Vergleich, Munich, 1988, vol. 3, p. 45-73.

22 «Étudier l'histoire grecque n'est donc pas la même chose pour nous qu'étudier l'histoire d'autres peuples. Les Grecs échappent complètement à la sphère de ces derniers [...] et nous méconnaissons tout à fait notre relation à eux si nous osons les juger à l'aune du reste de l'histoire universelle. Leur connaissance ne nous est pas simplement agréable, utile et nécessaire; ce n'est qu'en elle que nous trouvons l'idéal de ce que nous-mêmes désirons être et créer. Si toute autre partie de l'histoire nous enrichit en intelligence et en expérience humaine, nous puisons dans la contemplation des Grecs quelque chose qui n'est pas simplement humain (litt.: terrestre), mais presque divin.» W. v. HUMBOLDT, "Geschichte des Verfalls und des Untergangs der griechischen Freistaaten", in Gesammelte Schriften, éd. par A. LEITZMANN, Berlin, 1904 (réimpr. 1968), p. 188. Cf. CALVIÉ, “Antiquité et Révolution”, p. 455-475. 
L'idéal éducatif du "nouvel humanisme» (neuhumanistisches Bildungsideal) était d'abord, et en raison de ses origines, un idéal purement individualiste ${ }^{24}$. Ce n'est que lentement et très progressivement que l'idéalisme allemand s'est transformé au cours des années 1792 à 1817 , passant d'un rejet de l'État à une idéalisation de l'État. Pour Wilhelm von Humboldt, comme pour Johann Wolfgang Goethe, le but de l'éducation "grecque» était encore d'éduquer l'homme (moderne) pour en faire un citoyen du monde (Erziehung zum Weltbürger). Cet esprit universaliste s'opposait à la nouvelle idée nationale, contrairement à l'idéologie universaliste de la Révolution française qui n'était pas en contradiction avec l'idée nationale. Dans sa biographie de Goethe, en 1936, Walter Rehm exprime cette idée en ces termes:

«Der Mensch, nicht der Staat war dieser Zeit Merkzeichen des Griechischen. Die "Polis", die "Urbs" war das Letzte, was Goethe und nicht nur ihn an der Antike, am Griechentum fesselte. [...] Der Staat war in diesen deutschen Griechen-Mythos nicht aufgenommen.» 25

Dans le climat politique de l'époque, une telle tension entre la conception de l'Antiquité et l'idée de nation ne pouvait se maintenir très longtemps. Le début d'une nouvelle phase dans la relation à la Grèce antique peut être situé vers les années 1820 . Cette deuxième étape est celle de la neuhumanistische Gymnasialreform, de la réforme des lycées, en Bavière surtout, mais aussi en Prusse et ailleurs. Un des objectifs de ce courant réformiste était de relier, à partir du "nouvel humanisme», la sittliche Bildung der Griechen non plus à un Bildungsideal weltbürgerlicher Humanität, mais à l'ordre politique (bürgerliche Ordnung) de l'époque présente ${ }^{26}$.

Dès la première moitié du XIX ${ }^{\mathrm{e}}$ siècle, plusieurs tendances commencèrent, progressivement, à miner la base même du mythe grec: la philosophie de l'histoire de Hegel avait dévalorisé l'Antiquité en en faisant une étape préliminaire à la modernité, en en faisant une époque, dans le double sens du mot aufgehoben, à la fois préservée

24 RÜEGG, “Antike als Begründung”, p. 274, citant E. SPRANGER, Wilhelm von Humboldt und die Reform des Bildungswesens, Unveränderte Neuausgabe mit Nachträgen, Tübingen, 1960 [1910], p. 6 sv.: "Der deutsche Idealismus entwickelte sich in den Jahren 1792-1817 nur langsam von der Staatsfeindschaft zum Staatsidealismus.»

25 "Pour cette époque, ce fut l'Homme, et non l'État, le signe caractéristique de la grécité La polis, l'urbs [la cité], c'était la dernière chose qui intéressait Goethe, et pas seulement lui, dans la grécité. [...] L'État n'était pas intégré dans ce mythe grec allemand.», W.R EHM, Griechentum und Goethezeit. Geschichte eines Glaubens, Leipzig, 1936, p. 22 [4e éd. revue, Berne, 1969], cité par P. FUNKE, "Das antike Griechenland: eine gescheiterte Nation? Zur Rezeption und Deutung der antiken griechischen Geschichte in der deutschen Historiographie des 19. Jahrhunderts", in Annäherung an Griechenland. Festschrift für Anastasios Katsanakis zum 65. Geburtstag, éd. par H.-D. BLUME \& C. LIENAU (Choregia. Münstersche Griechenland-Studien, Heft 1), Münster, 2002, p. 81-101, ici p. 83.

26 Cf. RÜEGG, “Antike als Begründung”, p. 276. 
dans et abolie par l'époque moderne ${ }^{27}$. C'est grâce à l'Entfremdung (distanciation) à l'égard du monde immédiat, opérée par l'immersion dans l'Antiquité, que l'homme se construit comme être moral, capable de devenir patriote. Si cette immersion dans l'Antiquité grecque signifie, pour Hegel et le nouvel humanisme en général, un passage à travers un paradis aux capacités formatrices, il ne s'agit toutefois pas d'un paradis perdu, mais d'un monde concret et palpable à travers l'histoire, la littérature, la philosophie, l'art et les sciences. Éduquer et former des Allemands à travers cette immersion suppose par conséquent que le monde des Grecs devienne l'objet d'une science systématique de l'Antiquité (Altertumskunde) ${ }^{28}$. Ce tournant est en même temps le début du désenchantement de ce monde grec. Dans le discours cité au début, Wilamowitz souligne que " jene Antike, die ein absolut verbindliches Vorbild für Kunst und Leben sein sollte, war für beides eine ernste Gefahr, schon weil sie ein Wahnbild war.» 29

À partir de ces mêmes années, l'Altertumskunde chercha donc à saisir la particularité de l'Antiquité par le biais d'instruments et de méthodes scientifiques; elle découvrait les aspects concrets et quotidiens de l'Antiquité, sa normalité, et lui enlevait son caractère «idéal». Une tendance analogue se fit jour dans l'enseignement du grec qui devint de plus en plus, à partir du milieu du siècle, un exercice de logique appliquée, basé sur l'apprentissage de la grammaire. Le mythe grec perdait ainsi son fondement même dans les contenus de l'enseignement.

Walter Rüegg a analysé ce tournant dans le détail, en s'appuyant sur une lecture de nombreux écrits de réformateurs du système éducatif. Nous lui empruntons un exemple significatif; la transformation de la Grèce antique d'un mirage en un objet d'étude systématique, mais toujours dans un but éducatif, s'accompagna d'un recentrage, d'une redéfinition de l'objectif de cette éducation : l'Allemand "hellénisé» n'était plus le Weltbürger, mais un homme situé dans la réalité politique et sociale de son temps, dans le siècle de l'unité nationale. Ce changement de perspective rendait urgente une reformulation du rapport entre la Grèce et l'idée de nation, reformulation qui affectait également la position à l'égard de Rome et le choix entre Rome et la Grèce. Dans plusieurs articles de la grande Encyklopädie des gesammten Erziehungsund Unterrichtswesens, publiée entre 1859 et 1875 à l'intention des professeurs de l'enseignement secondaire, ces réorientations se traduisent par une mobilisation des valeurs grecques au profit de l'idée de nation, assortie d'un exercice d'équilibre entre la Grèce et Rome. Mais le souci de défense des orientations grecques reste

27 G.W.F. HEGEL, Vorlesungen über die Philosophie der Geschichte (Theorie Werkausgabe vol. 12), Francfort, 1970. Hegel a professé cinq fois, entre $1822 / 23$ et $1830 / 31$, son cours intitulé "Philosophie der Weltgeschichte".

28 Cf. RÜEGG, "Antike als Begründung", p. 278 sv.

29 WILAMOWITZ, "Weltperioden”, p. 130: "cette Antiquité, qui était censée être un modèle absolument obligatoire pour l'art et la vie, était pour les deux un danger sérieux, déjà parce qu'elle était une chimère.» 
prépondérant: l'auteur de l'article «Vaterlandsliebe» (patriotisme) explique ainsi aux enseignants que le patriotisme romain n'avait pas su dépasser la ville de Rome et les territoires régis par la res publica. Les Grecs, par contre, bien que divisés en plusieurs Stämme (tribus) étaient liés entre eux par une même origine et un même terroir ${ }^{30}$. «Et pour cette raison, le patriotisme grec comprend à la fois le don moral de soi à l'ordre étatique et l'amour naturel du sol de la patrie et des compatriotes." ${ }^{31} \mathrm{Le}$ formalisme du droit romain est également dévalorisé, tandis que l'on insiste sur le fait que «l'origine commune des Grecs et le territoire qu'ils partageaient l'emportaient sur la division en Stämme». L'analogie avec la théorie concomitante des médiévistes allemands d'une division en Stämme d'un peuple originel allemand-germanique uni au début de l'histoire n'était que trop évidente pour le public allemand des années 1850. La valorisation de la Grèce par rapport à Rome apparaît de même dans l'article «Nation». On y affirme qu'une éducation nationale au sens d'une éducation du peuple entier n'a existé que deux fois dans l'histoire: en Grèce d'abord, en Allemagne ensuite, depuis Fichte. La valeur éducative, formatrice de l'Antiquité, et en particulier de la "grécité» (Griechentum), était maintenant légitimée par le fait que l'idée de nationalité et l'idéal de sa fonction éducatrice y étaient nés ${ }^{32}$. Le Weltbürger du nouvel humanisme avait vécu.

\section{Et les historiens ?}

Dans le contexte du mouvement de libération contre Napoléon, le mythe d'une affinité élective entre la Grèce et l'Allemagne - mythe encore renforcé par le soutien enthousiaste que les nationalistes allemands apportèrent à la lutte de libération grecque contre l'Empire ottoman - avait pour conséquence naturelle, dans le traitement de l'histoire antique, que les historiens et philologues allemands prenaient parti pour Démosthène contre Philippe II de Macédoine. C'est ainsi que Niebuhr publia en 1805 , de manière anonyme, une traduction de la première Philippique de Démosthène et la dédia au tsar Alexandre ${ }^{33}$.

Pourtant, l'identification avec la Grèce des petites cités particulières, avec la Kleinstaaterei ne pouvait satisfaire à la longue les historiens de la nouvelle génération,

30 «Der römische Patriotismus sei auf die Stadt Rom und die von dort ausgehenden rechtlichen Ordnungen der res publica beschränkt gewesen. Die Griechen hingegen seien trotz der Geteiltheit in die verschiedenen Stämme durch die gleiche Abstammung und den gleichen Boden miteinander verbunden": cf. RÜEGG, "Antike als Begründung", p. 271.

31 "Und darum ist im griechischen Patriotismus mit der sittlichen Hingebung an die Ordnung des Staates auch die natürliche Anhänglichkeit an den vaterländischen Boden und an die Volksgenossen verbunden.»: cf. RÜEGG, “Antike als Begründung”, p. 271.

32 "Der Bildungswert des Altertums, insbesondere des Griechentums wird vor allem darin gesehen, daß dort die Idee der Nationalität erweckt und das Bildungsideal der Nationalität erweckt wurde»: cf. RÜEGG, "Antike als Begründung”, p. 272, avec référence à l'article «Bildungsideale» de l'encyclopédie.

$$
\text { Cf. FUNKE, "Das antike Griechenland", p. } 85 .
$$


engagés dans le combat pour l'unité allemande. Quand Wilhelm von Humboldt rédigea, à Rome, en l'hiver 1807-1808, la préface à une Geschichte des Verfalls und des Untergangs dergriechischen Freistaaten, un livre qu'il ne porta pas à terme, son objectif politique était encore la défense des libertés et du système fédéral, la défense de l'individualité face à une constitution politique qui la restreindrait nécessairement. Tant que, dans la discussion politique, le concept de nation n'était pas encore lié, de manière absolue, à celui d'État, l'idée d'une nation grecque, basée sur la conception d'une civilisation commune aux nombreux États particuliers et assurant la liberté politique ainsi que la prospérité culturelle, pouvait conforter les Allemands dans leur propre conception d'une Kulturnation. Mais cette analogie avec l'histoire grecque ne pouvait faire oublier la défaite des cités libres face à Philippe II de Macédoine lors de la bataille de Chéronée en 338 av. J.-C. 34

C'est Johann Gustav Droysen qui, dans son Histoire d'Alexandre le Grand, publiée en 1833 - l'auteur n'avait que vingt-cinq ans - sautera le pas et proposera aux patriotes allemands de changer de camp et de se rallier à Philippe II et à Alexandre contre la Grèce des cités. Alors que la première édition de son ouvrage s'ouvre, de façon plutôt traditionnelle, par une longue évocation de l'opposition entre la Grèce et l'Asie, la troisième édition, publiée, après l'unification allemande, annonce dès la première page le point de vue à partir duquel il envisage l'histoire grecque:

«Den alten Culturvölkern Asiens gegenüber sind die Hellenen ein junges Volk ; erst allmählich haben sich sprachverwandte Stämme in diesem Namen zusammengefunden ; das glückliche Schaffen einer nationalen, das vergebliche Suchen einer politischen Einheit ist ihre Geschichte.» 35

Droysen condamnait sans réserve la faillite misérable de la griechische Kleinstaaterei ${ }^{36}$ et prenait parti pour la puissance militaire des Macédoniens. Puisque les Grecs étaient incapables de réaliser, voire de vouloir eux-mêmes l'unité nationale, ils devaient y être contraints par l'État militaire de leurs «parents situés de l'autre côté

34 Cf. FUNKE, "Das antike Griechenland", p. 90.

35 J.G. DROYSEN, Geschichte Alexander des Großen, Gotha, $3^{\mathrm{e}}$ éd. 1880, p. 1, (traduction française: J.G. Droysen, Histoire de l'Hellénisme, traduite de l'allemand ss la dir. d'A.B OUCHÉ-LECLERCQ, Paris, 1883-1885). Comme dans l'édition allemande de 1877-1878, l'Histoire d'Alexandre le Grand forme le premier tome de la deuxième édition de l'Histoire de l'Hellénisme (cf. la réédition intégrale établie par P. PAYEN, Grenoble, 2005). La traduction française a été établie à partir de la $2^{\mathrm{e}}$ édition allemande dont le début est fort différent et commence par la phrase célèbre : «Der Name Alexander bezeichnet das Ende einer Weltepoche, den Anfang einer neuen.» ("Le nom d'Alexandre signifie la fin d'une époque de l'histoire mondiale, le début d'une nouvelle.»).

36 K. CHRIST, "Johann Gustav Droysen (1808-1884)", in ID., Von Gibbon zu Rostovtzeff. Leben und Werk führender Althistoriker der Neuzeit, Darmstadt, 1972, p. 50-67, p. 55. 
de la frontière» ${ }^{37}$. Dans une lettre du $1^{\mathrm{er}}$ septembre 1834 , adressée à F.G. Welcker, Droysen avait ainsi motivé son choix:

«Ich (bin) ein Verehrer der Bewegung und des Vorwärts: Cäsar, nicht Cato, Alexander und nicht Demosthenes ist meine Passion. [...] Weder Cato noch Demosthenes begreifen mehr die Zeit, die Entwicklung, den unaufhaltsamen Fortschritt.» 38

L'Histoire et Bismarck allaient donner raison à Droysen. Dans la deuxième édition de son Alexandre le Grand, parue en 1877, l'analogie entre l'unification de la Grèce par Philippe II et celle de l'Allemagne par la Prusse saute plus aux yeux que dans la première édition de $1833^{39}$. Mais ceci se situe après 1848 et 1871 et n'est donc pas très étonnant. Ce qui pourrait étonner - mais ne fait que confirmer la faiblesse ou la rareté du recours rhétorique et historique à l'Antiquité au sein du mouvement national allemand - c'est l'absence totale de référence à l'idée de l'unification de la Grèce par Philippe et Alexandre dans le grand discours que Droysen prononça en 1843, à l'occasion du millénaire du traité de Verdun. Le thème traité par Droysen était l'origine de l'Allemagne et son cheminement vers un État fondé sur le fait national ${ }^{40}$. On aurait pu s'attendre à ce que l'historien d'Alexandre le Grand mette sa conception de l'unification de la Grèce au service de sa vision de l'unité allemande. Mais cela ne correspondait apparemment pas à la situation des années antérieures à 1848. Par ailleurs, la comparaison entre la Macédoine et la Prusse n'était pas entièrement neuve ; elle avait été esquissée plusieurs fois déjà, en Angleterre, au XVIII ${ }^{e}$ siècle. Ce qui était nouveau et lourd de conséquences, c'était l'application de l'idée de Staatsnation au monde des États grecs de l'Antiquité ${ }^{41}$.

37 Cf. O. HintzE, "Johann Gustav Droysen”, in ID., Soziologie und Geschichte. Gesammelte Abhandlungen zur Soziologie, Politik und Theorie der Geschichte, éd. par G.O ESTREICH, Göttingen, 1964 (3 éd. 1982), p. 453-499, citation p. 461. P.PAYEN, “Droysen, l'Histoire d'Alexandre le Grand et l'État prussien: la querelle de l'unité et la question du "modernisme»", in S. CAUCANAS, R. CAZALS \& P. PAYEN (ss la dir. de), Retrouver, imaginer, utiliser l'Antiquité. Actes du colloque international tenu à Carcassonne les 19 et 20 mai 2000, Toulouse, 2001, p. 115-134.

38 "Je suis un fervent partisan du mouvement vers l'avant: César et non Caton, Alexandre et non Démosthène, sont ma passion. [...] Ni Caton, ni Démosthène ne comprennent plus leur époque, l'évolution, le progrès irrésistible.» Cité par FUNKE, "Das antike Griechenland", p. 91.

39 CHRIST, “Droysen”, p. 55, avec référence au débat entre A. Momigliano et B. Bravo sur l'importance des différences entre la $1^{\mathrm{e}}$ et la $2^{\mathrm{e}}$ édition.

40 J.G. DROYSEN, Rede zur tausendjährigen Gedächtnisfeier des Vertrages zu Verdun, Kiel, 1843. Réimprimé in F. STRICH, Deutsche Akademiereden, Munich, 1924, p. 89-110. Il n'y a que de très rares allusions générales à l'Antiquité dans ce discours.

41 FUNKE, "Das antike Griechenland", p. 91. Pour l'analogie entre la Prusse et la Macédoine, voir aussi l'article d'A. MAURY, "Une Prusse dans l'Antiquité", Revue des Deux Mondes (1 ${ }^{\mathrm{er}}$ novembre 1870), cf. F. HARTOG, Le XIXe siècle et l'histoire. Le cas Fustel de Coulanges, Paris, 1988, nouvelle édition, 2001, p. 61. 
Après l'échec de la révolution de 1848 et le refus de la couronne impériale par le roi de Prusse, Frédéric Guillaume IV, Droysen prit clairement position, dans une feuille volante publiée le 7 août 1849, contre certaines tendances du parlement de Francfort de vouloir fonder l'État national sur la seule force des idées nationales et libérales. Au contraire, il fallait qu'une puissance se décide à s'opposer aux autres puissances et à défendre l'Allemagne contre les égoïsmes particuliers de celles-ci. «La cause de la nation est maintenant entre les mains de la Prusse. [...] Être la puissance allemande est son destin.» Et ce destin implique, d'une part, la nécessité de quitter le terrain du droit et de l'ordre international tel qu'il avait été fondé en 1815 et, d'autre part, celle de restaurer la véritable idée impériale, qui avait été ruinée par la politique dynastique de l'Autriche depuis Charles-Quint ${ }^{42}$.

Droysen lui-même se détourna de l'histoire de l'époque hellénistique pour se consacrer à l'histoire de la Prusse. Sa Geschichte des Hellenismus, dont il publia le premier volume en 1836 et qu'il abandonna après la publication d'un deuxième volume en 1843, devenait ainsi, comme l'a dit Arnaldo Momigliano, une "praeparatio evangelica à l'histoire de la Prusse» ${ }^{43}$. Pourtant, ce serait méconnaître la véritable tâche politique que Droysen assigne à l'historien que de ne voir, dans ses réinterprétations du sens de l'histoire de la Grèce et de l'Allemagne, qu'une prise de position en faveur de l'unification allemande sous la direction de la Prusse. Pour Droysen, la «vision historique» du temps présent devait dépasser l'ordre de l'État national et prendre la mesure du tournant historique, d'ampleur comparable à celui de l'hellénisme, que signifiait l'ère nouvelle du capitalisme et du bonapartisme ${ }^{44}$.

La nouvelle conception de l'histoire grecque, par le biais de laquelle Droysen accordait à l'hégémonie acquise par le royaume de Macédoine la même légitimité que celle exercée successivement par Sparte, Athènes et Thèbes ${ }^{45}$, ne répondait pas seulement à des motivations politiques actuelles. Ce qui importait le plus à ses yeux,

42 «... Nicht von der "Freiheit", nicht von nationalen Beschlüssen aus war die Einheit Deutschlands zu schaffen. [...] Die Sache der Nation ist jetzt bei Preußen [...] Die deutsche Macht zu sein, ist seine geschichtliche Aufgabe [...] In diesem Sinne an die Spitze Deutschland tretend, erneuere uns Preußen die wahrhafte Idee des Kaisertums, wie sie seit dem fünften Karl an der dynastischen Politik Österreichs zugrundegegangen ist !» Cité par HINTZE, "Droysen”, p. 476; cf. O. HINTZE, "Johann Gustav Droysen und der deutsche Staatsgedanke im 19. Jahrhundert", in HINTZE, Soziologie und Geschichte, p. 500-518, ici p. 510.

43 A. MOMiGLiAnO, "J.G. Droysen entre les Grecs et les Juifs", in A. MOMIGLiANO, Problèmes d'historiographie ancienne et moderne, Paris, 1983, p. 383-401, citation p. 390.

44 J. RÜSEN, "Johann Gustav Droysen", in H.-U. WEHLER (éd.), Deutsche Historiker, vol. 2, Göttingen, 1971, p. 7-23, ici p. 14.

45 J.G. DROYSEN, "Vorwort zur "Geschichte des Hellenismus II"”, in W. NIPPEL (éd.), Über das Studium der Alten Geschichte, Munich, 1993, p. 128-147, ici p. 145. 
c'était de présenter l'hellénisme comme "die moderne Zeit des Altertums" (l'époque moderne de l'Antiquité) ${ }^{46}$.

La revalorisation radicale de l'époque hellénistique, proposée par Droysen, n'a pas emporté l'entière adhésion des antiquisants allemands de son temps, loin de là. L'Histoire grecque d'Ernst Curtius ${ }^{47}$, rédigée à partir des années 1850, en est la preuve la plus manifeste. On ne pouvait pas si vite renoncer à l'identification entre l'Allemagne et la Grèce de l'époque classique. Mais le problème était insoluble et, pour ne citer qu'un exemple, plus tardif, on perçoit bien le malaise de Wilamowitz qui, dans son Aristoteles und Athen (1893), reproche à Aristote d'avoir méconnu le caractère national (nationalstaatliche Natur) de l'empire athénien, d'avoir sous-estimé le «rôle national» des États fédératifs grecs (der griechischen Bundesstaaten) et d'avoir refusé de penser «l'unification de parties autonomes en un tout plus grand» 48 . À peu près à la même époque, Nietzsche, qui partageait avec la tradition allemande la dévalorisation des périodes hellénistique et romaine, opposa au culte de la Grèce classique une revalorisation de la période archä̈que. Pour lui, l'apogée de la Cultur der Polis devait être datée entre 776 et 560 av. J.-C. C'est à cette époque qu'on trouvait les véritables Grecs, aristocrates panhelléniques, vivant selon l'idéal de l'agôn. Ce n'est pas la cité évoluée de la période classique qui offrait, selon lui, les meilleures conditions pour atteindre l'apogée culturel, mais l'État «incomplet», embryonnaire de l'époque archaïque qui favorisait l'énergie et la force sauvage pouvant être investies dans les relations intellectuelles ${ }^{49}$.

46 DROYSEN, “Vorwort”, p. 145. Cf. P. PAYEN, "Droysen, l'Histoire d'Alexandre le Grand" insiste justement sur la volonté de Droysen de "comprendre comment les apports des conquêtes d'Alexandre ont pu se fondre dans l'héritage des cités grecques» (p. 120) et d'interpréter son action comme une tentative de "favoriser la "fusion" entre Orient et Occident» (p. 126).

47 Ernst Curtius (1814-1896) était philologue, historien et de plus en plus archéologue. De 1844 à 1849, précepteur du futur empereur Frédéric III, auteur de l' Histoire grecque en langue allemande la plus lue au XIX ${ }^{\mathrm{e}}$ siècle (première édition en 3 vol., 1852-1867), conçue par la maison d'édition Weidmann comme le pendant de l'Histoire romaine de Mommsen ; la proposition avait d'abord été faite à Droysen.

48 «Die vereinigung selbständiger glieder $\mathrm{zu}$ einem größeren ganzen $\mathrm{zu}$ verfolgen» (Aristoteles und Athen, I, p. 367), cité par CANFORA, " Politik" in der Wissenschaft", in CALDER, Wilamowitz, p. 56-79, citation p. 63. Dans un rapport rédigé pour une Schulkonferenz en 1900, Wilamowitz adopte la position de Droysen en déclarant que les élèves doivent connaître Alexandre le Grand, fondateur de la civilisation (Kultur) qui a donné naissance au christianisme et à l'organisation de l'Empire d'Auguste, et non Démosthène et ses discours éphémères. Cf. aussi la présentation plus détaillée dans CANFORA, "Wilamowitz und die Schulreform", p. 642.

49 Cf. l'analyse détaillée de H. CANCIK, Nietzsches Antike. Vorlesung, Stuttgart-Weimar, 1995, en particulier p. $98 \mathrm{sv}$. 
Les hellénistes de métier, comme Ernst Curtius, directeur des fouilles d'Olympie, ou Wilamowitz, maître incontesté des philologues ${ }^{50}$, n'étaient pas prêts à abandonner la Grèce classique, que ce fût au profit du roi de Macédoine ou à celui des États hellénistiques. Pourtant, la défense d'Athènes n'allait pas sans sacrifices. Dans un discours en l'honneur de l'empereur sur Die Entwicklung des preußischen Staats nach den Analogien der alten Geschichte, en 1882, Curtius concédait l'échec national de la cité de Périclès :

" Athen ist auf geistige Erfolge beschränkt geblieben und hat den Untergang des

Vaterlands nicht aufhalten können. Uns ist ein besseres Los gefallen. [...] Kaiser Wilhelm (hat) die Siege erfochten, durch welche unser Vaterland vor dem Schicksal Griechenlands bewahrt worden ist.» 51

Quelques années auparavant, Wilamowitz était allé plus loin. Dans son discours en l'honneur de l'anniversaire de l'empereur en 1877, il avait apostrophé les «nobles citoyens d'Athènes» en leur notifiant que «das beste», la chose la plus précieuse, leur avait manqué. Car l'amour suprême, mais abstrait, voué aux institutions, aux lois et aux idées ne pouvait rivaliser avec un dévouement à l'institution et à l'idée, porté par un véritable don de soi, de personne à personne, «à l'empereur allemand, notre roi» 52 .

La Grèce antique était ainsi devenue l'exemple d'une gescheiterte Nation, une nation qui avait échoué - et c'est ainsi qu'elle apparaîtra chez les historiens de la génération suivante, chez Georg Busolt (1850-1920), Julius Beloch (1854-1929) et Eduard Meyer (1855-1930) -, mais le nouvel État national allemand unifié n’eut pas de mal à trouver un autre terrain d'identification historique dans l'Antiquité grecque qui était restée néanmoins la référence principale des élites cultivées allemandes.

Ce nouveau terrain avait été préparé dès le lendemain de l'échec de la révolution de 1848, quand Ernst Curtius déclara, en 1852, que le site d'Olympie avait été «le centre de la vie nationale et de la conscience helléniques» (des hellenistischen Nationallebens und Bewußtseins) ainsi que de la cohésion nationale (des nationalen

50 Dans une lettre adressée en janvier 1845 à Max Duncker, Droysen avait traité les philologues de Ungeziefer, de "vermine", qui, au contraire des historiens, n'auraient aucune idée des défis ni de l'époque hellénistique, ni de l'époque contemporaine. Cité par FUNKE, "Das antike Griechenland", p. 89.

51 "Athènes a dû se contenter de succès intellectuels et culturels et n'a pu empêcher le déclin de la patrie. Nous autres Allemands avons connu un sort meilleur [...]. L'empereur Guillaume, en combattant victorieusement, a épargné à notre patrie le destin de la Grèce.» E. CURTIUS, "Die Entwicklung des preußischen Staats nach den Analogien der alten Geschichte", in Altertum und Gegenwart. Gesammelte Reden und Vorträge, vol. 2, Berlin, 1882, p. 207-218, citation p. 216, due à FUNKE, "Das antike Griechenland", p. 94. De la même teneur est le discours de Wilamowitz-Moellendorff à l'occasion de l'anniversaire de l'empereur en 1877 ("Von des attischen Reiches Herrlichkeit"), cité supra, p. 18 et note 5.

52 WILAMOWITZ, "Von des attischen Reiches Herrlichkeit", p. 63. 
Zusammenschlusses) ${ }^{53}$. Il chercha à enthousiasmer son public, et parmi lui le roi de Prusse, pour les fouilles d'Olympie en déclarant les Allemands "héritiers et propriétaires de la science et des arts fondés sur l'Antiquité classique». Mais il fallut attendre la fondation du Reich pour obtenir un véritable et solide soutien aux campagnes archéologiques à Olympie et sur d'autres sites. Bernd Sösemann a récemment analysé la manière dont la presse officielle et privée a été utilisée pour populariser les campagnes de fouilles, pour en faire un objet de fierté et de propagande nationales, dans un climat de concurrence avec les autres nations. Le mythe grec fut ainsi recentré sur Olympie. Dans la revue Im neuen Reich, on pouvait lire, en 1876, que les efforts des chercheurs allemands en Grèce, et tout particulièrement les résultats de leur travail à Olympie, témoignaient du fait,

«daß durch die Aufrichtung des Reiches unserem Volke erst die Möglichkeit eröffnet wurde, auch Friedenswerke und Unternehmungen der Wissenschaft, anderen Völkern gleich, im großen Stile anzufassen.» 54

La Reichs-Grabung Olympia, les fouilles entreprises par Curtius, Dörpfeld et d'autres au nom du Reich, était considérée par les archéologues et par le pouvoir politique comme un véritable National-Denkmal, un monument national. La primeur de l'annonce des progrès des fouilles était réservée au Reichsanzeiger, au bulletin officiel du Reich, avant d'être repris par l'ensemble de la presse nationale, régionale et locale. Dans ses multiples rapports et conférences, Curtius présentait les fouilles comme «das nationale Werk, an welchem vom Kaiserhause bis zum Bauernhofe alle guten Deutschen frohen Anteil nehmen.» 55 Face à la tare originelle du Reich, dont la fondation avait été obtenue par la force et qui avait été proclamé à l'étranger, et face aux difficultés internes, «l'œuvre de paix» des archéologues et historiens devait rattacher le nouvel État à la grande tradition grecque du XIXe siècle allemand, renforçant ainsi sa légitimité et contribuant à l'intégration de ses diverses composantes.

53 Cité par B. SÖSEMANN, "Olympia als publizistisches National-Denkmal. Ein Beitrag zur Praxis und Methode der Wisssenschaftspopularisierung im Deutschen Kaiserreich”, in H. KYRIELEIS (éd.), Olympia 1875-2000. 125 Jahre Deutsche Ausgrabungen, Mainz, 2002, p. 49-84, ici p. 51. Pour une étude détaillée du rôle de l'Antiquité grecque, et en particulier de l'archéologie, cf. S.L. MARCHAND, Down from Olympus. Archeology and Philhellenism in Germany, 1750-1970, Princeton,1996.

54 "... que c'est seulement l'établissement de notre Empire qui a ouvert à notre peuple la possibilité d'entreprendre, à l'égal d'autres peuples, des œuvres de paix et des opérations scientifiques de grande envergure», cité par S ÖSEMANN, "Olympia”, p.57.

55 «... l'œuvre nationale à laquelle tous les bons Allemands, de la maison impériale jusqu’à la ferme du paysan, accordent d'un cour joyeux leur intérêt», cité par SÖSEMANN, "Olympia”, p. 71. Cf. également A.H. BORBEIN, "Klassische Archäologie in Berlin vom 18. bis zum 20. Jahrhundert”, in W. ARENHÖVEL \& Ch. SCHREIBER (éd.), Berlin und die Antike, Berlin, 1977, p. 99 sv. pour les rapports entre la fondation du Reich et les fouilles d'Olympie. 
À côté de la mobilisation, aux alentours de 1871, des références historiques médiévales, notamment par le biais de la construction d'une généalogie toute fictive, reliant le nouveau Deutsches Reich à un Empire allemand médiéval qui n'avait jamais existé 56 , la part de l'Antiquité grecque dans les constructions identitaires officielles, ce qu'on appelle aujourd'hui la Geschichtspolitik ou Erinnerungspolitik, ne doit pas être surestimée. Cependant, son importance pour le Bildungsbürgertum allemand ne doit pas non plus être sous-estimée. En cela, elle dépassait largement le cadre des " fouilles impériales ", seul domaine, en définitive, où les objectifs des antiquisants et ceux des milieux politiques officiels se rencontraient véritablement.

\section{L'histoire romaine, les Germains et l'unité allemande}

Tout au long de la première moitié du XIX ${ }^{\mathrm{e}}$ siècle, et même au-delà, l'opposition entre Grecs et Romains avait été l'écho et l'amplificateur du sentiment anti-français ou anti-napoléonien du mouvement unitaire allemand. Mais le rejet de la référence romaine, parce que française, n'allait pas résister très longtemps aux réalités politiques. Le rapprochement entre la situation allemande et l'unification de l'Italie antique, puis contemporaine devait s'imposer comme une évidence. À la différence, pourtant, de la liberté grecque que l'on avait pu invoquer contre l'envahisseur français, l'histoire romaine n'allait pas servir de mobilisateur pour le mouvement d'unité. Elle n'y est apparue qu'après l'échec de la révolution de 1848, sous la plume de Theodor Mommsen (Römische Geschichte, en trois volumes, 1854-1856), fervent défenseur des idées libérales. Chez Mommsen, ce n'est pas l'histoire allemande qui est lue à travers l'histoire romaine, mais plutôt l'inverse. Comme le dira plus tard le plus grand admirateur de César, Friedrich Gundolf:

«Il [Mommsen] ne projette pas l'autrefois sur l'aujourd'hui, non la sagesse clarifiée des mondes anciens sur son monde à lui : il transporte sur une Antiquité inoubliable sa propre souffrance, sa propre ardeur. [...] Son Histoire romaine [...] est contemporaine à un degré inouï. Elle est un bloc de tempérament.» 57

Dans l'Histoire romaine, l'on croyait pouvoir lire le soutien, avant la lettre, de Mommsen à Bismarck. Comme l'écrivait Gaston Boissier en 1865:

«Comme il est toujours préoccupé du présent dans ses études du passé, on dirait qu’il poursuit dans l'aristocratie romaine les hobereaux de la Prusse et qu'il salue d'avance

56 Voir H. BRUHNS, "La fondation de l'État national allemand et la question des origines", à paraître.

57 F. GUNDOLF, "Le XIX siècle et César", in F. GUNDOLF, César. Histoire et légende. Traduit de l'allemand par M. BEAUFILS, Paris 1933, p. 284 (éditions originales: F.G UNDOLF, Caesar. Geschichte seines Ruhms, Berlin, 1924; ID., Caesar im neunzehnten Jahrbundert, Berlin, 1926). 
dans César ce despote populaire dont la main ferme peut seule donner à l'Allemagne son unité.» 58

Le César de Mommsen, dans le troisième volume, paru en 1856, n'avait pas d'abord été lu dans la cadre de l'histoire allemande, mais dans la perspective de Napoléon III, dans le contexte du débat sur le bonapartisme et le césarisme, de sorte que Mommsen se voyait contraint de s'en expliquer dans la $2^{\mathrm{e}}$ édition de ce troisième volume en 1857:

«die Geschichte Caesars und des römischen Caesarentums ist bei aller unübertroffenen Großheit des Werkmeisters, bei aller geschichtlichen Notwendigkeit des Werkes, wahrlich eine bittrere Kritik der modernen Autokratie, als eines Menschen Hand sie zu schreiben vermag. [...] jede noch so mangelhafte Verfassung, die der freien Selbstbestimmung einer Mehrzahl von Bürgern Spielraum läßt, ist unendlich mehr als der kunstvollste und humanste Absolutismus ; denn jener ist der Entwicklung fähig, also lebendig, dieser ist, was er ist, also tot.» 59

Certes, l'on trouve Mommsen et Droysen au moins partiellement dans les mêmes camps politique et scientifique; mais il serait erroné, comme l'a montré Alfred Heuss, de compter le Mommsen de l'Histoire romaine parmi les historiens "politiques» 60. À la différence de Droysen qui a largement contribué à créer la légende de la mission allemande, unificatrice, de la Prusse, Mommsen n'a pas versé dans le Borussismus et, dans les années postérieures à 1871, il a été l'un des critiques les plus féroces de Bismarck. S’il est vrai que, dans la Rome républicaine de Mommsen, se poursuivaient, après l'échec de la révolution de 1848, les combats de l'assemblée nationale de Francfort et que s'y reflétait la lutte pour les libertés de la bourgeoisie allemande ${ }^{61}$, on peut difficilement parler d'une utilisation, voire d'une instrumentalisation de l'Histoire romaine par le mouvement national ou par l'État national. Certes, la perspective tout à fait originale de Mommsen aurait pu s'y prêter; il affirme d'entrée de jeu que son histoire romaine sera une histoire de l'Italie, de l'unification étatique de l'ensemble des peuples italiques:

«Es ist die Geschichte Italiens, die hier erzählt werden soll, nicht die Geschichte der Stadt Rom. Wenn auch nach formalem Staatsrecht die Stadtgemeinde von Rom es war, die die Herrschaft erst über Italien, dann über die Welt gewann, so läßt sich doch dies

58 Cité par RÜEGG, “Die Antike als Begründung”, p. 268.

59 "... l'histoire de César et du césarisme romain est, nonobstant la grandeur inégalée du maître d'œuvre et malgré la nécessité historique de l'œuvre, en vérité une critique plus amère de l'autocratie moderne que la main d'un homme ne pourrait l'écrire.[...] toute constitution, aussi imparfaite soit elle, qui accorde de l'espace à la libre autodétermination par une majorité de citoyens, vaut infiniment plus que l'absolutisme le plus ingénieux et le plus humain; car celle-là est capable d'évoluer, donc vivante, celuici est ce qu'il est, donc mort", cité par A.W UCHER, "Theodor Mommsen", in H.U.W EHLER (éd.), Deutsche Historiker, vol. 4, Göttingen, 1972, p. 7-24, citation p.21.

60 A. HEUSS, Theodor Mommsen und das 19. Jahrhundert, Kiel, 1956, p. 335 sv.

61 S. REBENICH, Theodor Mommsen. Eine Biographie, Munich, 2002, p. 91. 
im höheren geschichtlichen Sinne keineswegs behaupten und erscheint das, was man die Bezwingung Italiens durch die Römer zu nennen gewohnt ist, vielmehr als die Einigung zu einem Staate des gesamten Stammes der Italiker, von dem die Römer wohl der gewaltigste, aber doch nur ein Zweig sind.» 62

Cette dévalorisation de Rome faisait écho aux idées politiques du jeune journaliste Mommsen qui, en 1848, avait plaidé pour que la Prusse se fondît dans l'Allemagne. Dans les années 1860, il se ralliera à Bismarck, avant de devenir, une décennie plus tard, un de ses adversaires les plus déterminés. Pour Mommsen, la construction de la Nation constituait tout simplement le sens de l'Histoire ${ }^{63}$ et l'on rappelle souvent que le Latium était pour lui «le prototype du développement national». Quant à la question du développement national en Grèce, Mommsen ne rejoint pas la perspective de Droysen. Il est plus proche du nouvel humanisme, car il précise, ce que l'on ne cite pas toujours:

«[...] wenn [...] Hellas der Prototyp der rein humanen, so ist Latium nicht minder für alle Zeiten der Prototyp der nationalen Entwicklung ; und wir Nachfahren haben beides zu verehren und von beiden zu lernen.» 64

Il ne s'agit là nullement d'une concession rhétorique à la grécomanie allemande, car Mommsen reprend cette idée avec force à la fin de sa Römische Geschichte qui culmine et se termine avec César, un personnage qui réunit en lui-même et dans ce qu'il a créé ou projeté «die beiden großen Wesenheiten des Menschentums, die allgemeine und die individuelle Entwicklung oder Staat und Kultur» ${ }^{65}$. On est loin

62 Th. MOMMSEN, Römische Geschichte, vol. 1 (9e éd., Berlin, 1902), p. 6 (= réimpression Munich, 1976, vol. 1, p. 22). Traduction française: Histoire romaine, Paris, réédition dans la collection Bouquins, Paris, 1985, vol. 1, p. 19: "C'est l'histoire de l'Italie et non pas l'histoire de Rome que nous voulons raconter. À ne regarder que les apparences du droit public formel, la cité de Rome a conquis d'abord l'Italie, puis le monde. Il n'en est point ainsi dans un sens historique plus élevé. Ce qu'on appelle la domination de Rome sur l'Italie est bien plutôt la réunion en un seul État de l'ensemble du peuple italique (des gesamten Stammes der Italiker), parmi lequel sans doute les Romains sont les plus puissants, mais sans être autre chose qu'une branche de la souche commune.» (traduction revue par H.B.).

63 Cf. A. HEUSS, Theodor Mommsen, p. 85: «Die Nation und der Nationalstaat sind für Mommsen keine geschichtlichen Bildungen und damit in den Fluß der Zeit hineingestellt, sondern naturgesetzliche Daten, mit denen er nicht nur normativ als zeitlosen Maßstäben rechnet, sondern die er auch ohne Bedenken in der Geschichte als Realitäten ansetzt.»

64 «Si la Grèce représente le prototype de l'évolution purement humaine, le Latium restera pour toujours le prototype du développement national; et nous, leurs descendants, devons les vénérer l'une et l'autre et nous inspirer de tous les deux.» MOMMSEN, Römische Geschichte, vol. 1, p. 176 (= réimpr. 1976, vol. 1, p. 190). Pour une analyse de la Römische Geschichte, cf. aussi C. MEIER, "Das Begreifen des Notwendigen. Zu Theodor Mommsens Römischer Geschichte”, in R. KOSELLEK, H. LUTZ \& J. RÜSEN, Formen der Geschichtsschreibung (Theorie der Geschichte, Beiträge zur Historik, vol. 4), Munich, 1982, p. 201-244.

65 MOMMSEN, Römische Geschichte, vol. 3, p. 568 (= réimpr. 1976, vol. 4, p. 233). 
de "César ce despote populaire dont la main ferme peut seule donner à l'Allemagne son unité» (G. Boissier).

L'énorme succès de l'Histoire romaine a détourné, pour un temps, l'intérêt du public cultivé du monde hellénique vers le monde romain et ses luttes de pouvoir, et l'on peut considérer à juste titre la Römische Geschichte comme un «welthistorischer Subtext zur Reichsgründungsära», comme une narration d'histoire universelle qui sous-tend l'époque de la fondation du Reich ${ }^{66}$. Pourtant, le César de Theodor Mommsen, "der erste und doch auch der einzige Imperator Caesar» ${ }^{67}$, ne pouvait être mis au service de la cause nationale allemande, et moins encore au service de Bismarck. Dans un certain sens, l'histoire romaine s'arrêta avec César. Mommsen luimême était incapable de passer de César à Auguste, voire même d'aller vraiment audelà de la bataille de Thapsus (46 av. J.-C.). Le principat et le römische Kaiserreich ne fournissent aucun élément utilisable pour le Reich allemand et son Kaiser. Le Reich de 1871 cherchera ses origines dans la fiction, élaborée par des historiens au milieu du siècle, d'un empire allemand médiéval. L'histoire romaine ne sera pourtant pas entièrement absente des constructions identitaires des années 1860-1870; mais il s'agira d'une histoire fondamentalement différente.

C'est d'abord l'histoire d'un délai et d'un avenir accordés par César à la civilisation classique. Sans l'abolition du régime sénatorial par César, dit Mommsen, les deutsche Stämme, dans lesquels César avait reconnu le véritable ennemi capable d'affronter le monde gréco-romain, auraient déferlé sans attendre sur l'Italie et ne lui auraient pas laissé le temps nécessaire pour porter la civilisation gréco-italique dans les provinces occidentales 68 .

C'est ensuite l'histoire d'un retour de Rome et de l'Italie, par le biais des Germains, dans les rêves identitaires allemands à l'époque de la fondation du Reich. Non en tant que vaincus occasionnels, à travers le souvenir de la victoire d'Arminius (qui avait déjà été mobilisé contre Napoléon Ier), sur les légions de Quinctilius Varus en l'an 9 de notre ère. Les princes allemands et les autorités publiques se méfiaient de l'enthousiasme populaire pour Arminius et retardaient la construction du monument en son honneur, commencée seulement en 1841 et achevée plus de trente ans plus tard. Entre-temps, l'idée populaire, mais politiquement indésirable, qu'il fallait trouver un nouvel Arminius parmi les princes allemands avait fait place à l'image d'une symbiose entre Arminius-Hermann et le peuple germanique-allemand. L'enthousiasme pour ce chef germanique, ancien soldat et citoyen romain, connut un renouveau spectaculaire après la victoire sur la France de Napoléon III, renouveau

66 G. HÜBINGER, Theodor Mommsen und das Kaiserreich, (Friedrichsruher Beiträge, vol. 22), Otto-von-Bismarck-Stiftung, Friedrichsruh, 2003, p. 19 sv.

67 MOMMSEN, Römische Geschichte, vol. 3, p. 569 (= réimpr. 1976, vol. 4, p. 234). Il s'agit de la dernière phrase de l'histoire romaine proprement dite, avant les parties consacrées aux arts, aux provinces, etc.

68 MOMMSEN, Römische Geschichte, vol. 3, p. 300 (= réimpr. 1976, vol. 4, p. 292). 
matérialisé par l'achèvement de la construction de l'imposant monument (Hermannsdenkmal) dans la forêt de Teutoburg en Westphalie, inauguré en 1875; les Hohenzollern et Bismarck manifestèrent alors un intérêt temporaire pour ce culte d'Arminius ${ }^{69}$. La fierté nationale y trouvait une cause commode: non pas tant par l'identification avec les Germains qui avaient écrasé l'armée romaine de Quinctilius Varus dans des forêts marécageuses, que par l'évocation des guerres de libération contre les Français: la statue monumentale est tournée vers l'ouest, vers la France, et non vers Rome. Mais cet épisode lointain de la confrontation entre Rome et les peuples germaniques ne pouvait être un élément historique central dans la construction d'une identité nationale; on n'allait pas, pour la seule idée de la «liberté» des Germains, renoncer à l'héritage de l'empire romain et du christianisme dans la préhistoire de ce qui aura été la grande affaire des historiens du XIXe siècle, l'invention d'un empire médiéval allemand. Dans cette dernière perspective, la fin de l'empire romain et les constructions «étatiques» des Germains sur son sol se prêtaient mieux que l'épisode d'Arminius à une connexion entre l'histoire romaine et «allemande».

Le retour des anciens Germains se manifesta de plus en plus nettement dans les années 1870, comme le montre de façon emblématique le cycle romanesque intitulé Die Ahnen (Les Ä̈eux) du libéral Gustav Freytag, écrit entre 1872 et 1880. Suggérant la continuité généalogique d'une famille allemande depuis le IVe jusqu'au XIXe siècle, à travers une cinquantaine de générations, l'auteur construit une biographie du peuple allemand dans laquelle la période de la liberté des villes, à partir du XII e siècle, apparaît comme une renaissance de la vie allemande, de la liberté que les AllemandsGermains avaient perdue sous les Mérovingiens et les Carolingiens ${ }^{70}$. La bourgeoisie comme peuple et nation est une constante de cette vision de l'histoire du libéralisme allemand.

Dans sa recherche de légitimité historique, le nouveau régime impérial avait projeté ses origines en arrière dans le temps jusqu'aux Hohenstaufen, voire même jusqu'au couronnement d'Othon le Grand en 962. Le roman historique permettait d'aller plus loin encore. Si le cycle romanesque Die Ahnen de Gustav Freytag valorisait surtout les libertés urbaines du Moyen Âge allemand, un autre roman, Ein Kampf um Rom (Une lutte pour Rome) de Felix Dahn, allait réorienter les rêves identitaires des Allemands sur l'Italie à l'époque de la guerre des Goths. C'est un fait étrange - mais qui au fond ne fait que refléter le décalage du Kaiserreich de 1871 par rapport aux rêves du mouvement d'unité antérieur à 1848 - que le grand roman historique des années de l'unité allemande ait été celui qui faisait des Goths les ancêtres des Allemands et qui projetait leur véritable destin dans une symbiose entre les Goths/Allemands et l'Italie. Dahn (1834-1912) était professeur d'histoire du droit

69 DOYE, “Arminius", p. 597 sv.

70 Cf., à propos de ce roman, R. KIPPER, Der Germanenmythos im Deutschen Kaiserreich. Formen und Funktionen historischer Selbstthematisierung, Göttingen, 2002, p. 95-109. 
allemand, auteur d'un grand nombre de romans historiques ainsi que d'ouvrages d'histoire ${ }^{71}$. Publié en 1876, Ein Kampf um Rom avait commencé à être rédigé en 1858 , dans le contexte de l'après 1848 et de la question italienne. Ce fut un des plus grands succès éditoriaux: cinquante éditions jusqu'en 1908, cent cinquante jusqu'en 1923, deux cent vingt jusqu'en 1930. Ce roman, qui narre l'histoire de la chute de l'empire ostrogoth en Italie, entre la mort de Théodoric (Flavius Theoderichus rex) en 526 et la bataille du Vésuve en 553, est devenu «l'exemple même d'un lieu de mémoire allemand» ${ }^{72}$. Dahn «réussit à faire, pour des générations d'écoliers, de cette branche des peuples germaniques, disparue sans héritiers [les Goths], les ancêtres par adoption des Allemands modernes qui ne sont en fait que leurs lointains petitscousins» ${ }^{73}$. C'est l'histoire d'une défaite héroïque, l'histoire de l'échec de l'établissement en Italie des Goths/Germains/Allemands - l'auteur passe sans cesse d'un terme à l'autre - , l'histoire de la lutte d'un peuple sans État contre un État sans peuple (Byzance). L'échec aussi du projet du héros du roman, le jeune roi Totila, d'acculturer les Goths en Italie ${ }^{74}$, de créer un nouveau peuple à partir d'une symbiose entre Goths/Allemands et Italiens, reflet du projet de Théodoric d'élever le Gothorum populus avec le populus Romanus (uterque populus) comme peuple dominant au-dessus des gentes de l'Occident ${ }^{75}$.

Ce roman volumineux est le travail à la fois d'un romancier et d'un historien qui cite ou paraphrase de longs passages des sources antiques, surtout de la Guerre des Goths de Procope. Dans la scène finale du roman, l'histoire cède le pas au rêve politique et historique: après la mort héroïque du roi Téja, les Goths, au lieu d'être anéantis, peuvent quitter l'Italie grâce à l'intervention des Nordlandhelden, les héros du pays du Nord, qui ramènent les «derniers Goths» et les corps des rois Théodoric et Téja vers Thuleland, la légendaire Thulé. Le lecteur comprend, comme l'a bien dit Arnold Esch, qu'ils reviendront, avec Charlemagne, avec Othon Ire, avec Barberousse

$71 \quad$ Felix DAHN publiera entre 1881 et 1889 une Urgeschichte der germanischen und romanischen Völker en 4 volumes.

72 "Formel eines deutschen Erinnerungsortes»: A. ESCH, “Ein Kampf um Rom”, in E.F RANÇOIS \& H. SCHULZE (éd.), Deutsche Erinnerungsorte, vol. 1, p. 27-40, citation p. 32. Cf. également P.U. HOHENDAHL, "Bürgerliche Literaturgeschichte und nationale Identität. Bilder vom deutschen Sonderweg", in KOCKA, Bürgertum, vol. 3, p.200-231.

73 J. ROVAN, Histoire de l'Allemagne. Des origines à nos jours, Paris, 1994, p. 39.

74 Voir à ce propos: J. SPIELVOGEL, "Die historischen Hintergründe der gescheiterten Akkulturation im italischen Ostgotenreich (493-553 n. Chr.)", Historische Zeitschrift 274 (2002), p. 1-24. Cf. K.F. WERNER, "Volk, Nation, Nationalismus, Masse: III. Mittelalter, IV. Volk/Nation als politischer Verband, V. Volk als Masse, Unterschicht", in Geschichtliche Grundbegriffe. Lexikon zur politisch-sozialen Sprache in Deutschland, éd. par O. BRUNNER, W. CONZE \& R. KOSELLECK vol. 7, Stuttgart, 1992, p. 171-281, cf. p. 188 à propos de la «römisch-gotische Symbiose».

75 Cassiodore, Variae 1, 1 (508); cf. 7, 3 (526). Cf. WERNER, "Volk, Nation ...", p. 189 et SPIELVOGEL, "Die historischen Hintergründe". 
mener un nouveau combat pour Rome ${ }^{76}$. En 1866, Felix Dahn regretta la défaite autrichienne et appela de ses vœux le Barberousse du poème de Rückert, c'est-à-dire l'empereur légendaire qui incarnait l'idée d'une grande Allemagne incluant l'Autriche ${ }^{77}$. Après la bataille de Sedan, Dahn substitua dans un poème qui eut un grand succès, "Barbablanca", qui était devenu le surnom de l'empereur GuillaumeI er, à "Barbarossa», rattachant le nouvel imperator directement aux anciens Germains. Dans la " petite " Allemagne de 1871, Ein Kampf um Rom donnait aux Allemands, et surtout à la jeune génération, une vision étonnante de leurs origines, de leurs racines et d'un destin très éloigné des réalités du nouvel État. C'était une façon de réclamer l'héritage de l'empire romain, de réunir en un nouveau peuple les valeurs et les qualités des Germains et des Romains. De telles idées n'étaient point réservées à la fiction romanesque. Ernst Curtius, dans un long discours intitulé «Rom und die Deutschen", prononcé en 1868 à l'université de Göttingen, les exprime pratiquement de la même manière. Dans les rapports si étroits et particuliers entre les Allemands et Rome, il n'y a pas la moindre place pour l'histoire romaine entre «le premier fait historique accompli par les Allemands (sic!)», qui était une expédition vers l'Italie ${ }^{78}$, et la guerre des Goths (p. 322). Dans ce discours de Curtius, l'Antiquité classique n'apparaît qu'à travers Winckelmann - c'est le même topos que dans le discours de Droysen en 1843 - et les savants allemands qui se rendent sur ses traces à Rome pour y découvrir, grâce à la supériorité de leur "savoir grec» (p. 331) l'art gréco-romain dans son unité et pour y fonder une nouvelle science de l'Antiquité. À Rome aussi, les Allemands sont des Grecs: «sie [die Deutschen] sind das priesterliche Volk, welches berufen ist, in reinen Händen die ewigen Güter der Menschheit zu tragen.» (p.336) 79 .

Le phantasme d'une symbiose entre les mondes germanique et romain était si éloigné des réalités et des problèmes auxquels le nouvel État et la société allemande -et aussi les différents États allemands ${ }^{80}$ - se voyaient confrontés au même moment,

76 ESCH, “ Ein Kampf um Rom”, p. 40.

77 Cf. A. BORST, "Barbarossa 1871", in A. BORST, Reden über die Staufer, FrancfortBerlin-Vienne, 1978, p. 91-178, p. 150.

78 Curtius ne précise pas à quelle expédition il pense; mais il s'agit très certainement de celle des Cimbres et des Teutons qui défirent les Romains en Norique, envahirent la Gaule et l'Espagne, avant que Marius ne leur barre la route de l'Italie en les battant à Aquae Sextiae (Aix-en-Provence) en 102 et à Vercellae (Verceil) en 101 av. J.-C.

79 E. CURTIUS, "Rom und die Deutschen. Rede, gehalten bei der jährlichen Preisverteilung in der Aula der Georgia Augusta-Universität in Göttingen am 4. Juni 1868", in Das Buch deutscher Reden und Rufe. Aus vier Jahrhunderten, éd. par A.K IPPENBERG \& F. v. d. LEYEN, Leipzig, 1943, p. 321-336.

80 Les différences régionales n'ont pu être abordées dans le cadre de cet article. Voir à ce sujet dernièrement: S. WEICHLEIN, Nation und Region. Integrationsprozesse im Bismarckreich (Beiträge zur Geschichte des Parlamentarismus und der politischen Parteien, 137), Düsseldorf, 2004. 
qu'on peut le lire plutôt comme une fuite devant la réalité de cette Allemagne prussienne que comme une contribution à la construction, a posteriori, d'une tradition historique pour l'État national allemand. Le grand succès des romans Die Ahnen de Gustav Freytag et Ein Kampf um Rom de Felix Dahn dans les années postérieures à 1871 s'explique sans doute d'abord par leurs qualités littéraire et romanesque intrinsèques. Mais il était lié, comme l'évocation croissante du passé germanique vers la fin du XIXe et le début du XXe siècle, à l'insatisfaction du sentiment national par rapport aux espoirs qu'avait nourris le mouvement national et unitaire avant 1871 . Le passé germanique était tellement lointain et indéfini que des traditions historiques et régionales fort différentes pouvaient s'en accommoder ${ }^{81}$. Le recours à une idéologie de la "germanité», à une conception substantialiste de l'ethnicité, le recours au potentiel intégrateur de l'époque germanique a été un phénomène postérieur à la fondation du Reich, et il a été une des réponses aux difficultés de réaliser la innere Staatsbildung. Pour le mouvement national allemand de la première moitié du XIX siècle, par contre, les anciens Germains n'avaient pas présenté un très grand intérêt.

En 1870, Fustel de Coulanges opposa, pour sa part, l'origine latine de la France à l'origine germanique de l'Allemagne:

«Nous sommes pour toujours des Latins par l'esprit. On peut donc laisser aux Allemands, avec leur Hermann, la gloire douteuse d'avoir refusé Rome, c'est-à-dire la civilisation.» 82

Si ce n'est qu'au moment de la fondation du Reich, les Allemands étaient -encore - des Grecs, et non des Germains, et qu'ils le resteront encore pour un certain temps ${ }^{83}$. Un demi-siècle plus tôt, Hegel avait répondu par avance à Fustel, et aussi à Fichte et Herder: "Wir wollen die Germanen nicht in ihre Wälder zurückverfolgen, noch den Ursprung der Völkerwanderung aufsuchen.» (Nous ne voulons pas suivre les traces des Germains jusque dans leurs forêts, ni chercher l'origine des grandes migrations). En dépit de l'image nostalgique de la Germanie peinte par Tacite, il ne fallait pas répéter, disait Hegel, l'erreur de Rousseau et considérer l'état sauvage comme un état "élevé» («hoher Zustand»), «in welchem der Mensch im Besitz der wahren Freiheit sei.» 84

81 Cf. KIPPER, Germanenmythos, p. 16, 75.

82 Cité par NICOLET, Fabrique, p. 277. Cf. HARTOG, Le XIX $X^{e}$ siècle et l'histoire.

83 Ainsi en 1886, dans un discours au titre évocateur de Basileia, à l'occasion du $25^{\mathrm{e}}$ anniversaire du gouverment "de sa Majesté notre empereur et roi», Wilamowitz regrette que le langage public formel (öffentliche Formensprache) se soit développé à partir du latin et non du grec, et il affirme, à propos de la royauté et de l'administration autonome des villes grecques (Selbstverwaltung der griechischen Gemeinde): "Das Hellenische stellt sich hier wie so oft dem Germanischen zur Seite, zu Römern und Romanen im Gegensatz.» («L'élément grec côtoie ici, comme si souvent, l'élément germanique, en opposition aux Romains et aux Latins»): Reden und Vorträge, p. 70 sv.

84 G.W.F. HEGEL, Vorlesungen über die Philosophie der Geschichte. (Theorie Werkausgabe, 12), Francfort, 1970, p. 419. Trad. fr.: Leçons sur la philosophie de l'histoire, traduit par J.G IBELIN, Paris, $3^{e}$ éd. remaniée, 1967. 


\section{Conclusions}

Au cours de ce siècle qui séparait Bismarck et Guillaume II de Goethe, la question du passé «national» et des origines de l'État allemand avait pris une importance de plus en plus grande. Le conflit entre des traditions antagonistes du Reich, les avatars du mouvement unitaire démocratique et la solution apportée par la force avaient pour corollaire que des traditions multiples et inconciliables furent mobilisées. Cette pluralité de traditions était l'expression paradoxale d'un manque de tradition. La nouvelle entité allemande n'avait pu s'inscrire dans aucune tradition existante, ni su en construire une qui aurait permis aux Allemands de s'identifier à ce nouvel État. Le grand roman de l'unité allemande, Ein Kampf um Rom, ne pouvait que détourner les aspirations de la jeunesse de l'État qui était le leur.

On peut distinguer, avec Helmut Plessner deux types de quêtes des origines d'un État: d'une part celle d'une origine idéelle, bien que fictive, que l'existence civique accorde à l'homme dans sa liberté, d'autre part celle d'une origine réelle, bien que mythique, qui se perd dans l'obscurité des temps anciens. Le rejet de Rome et l'idéal anti-étatique, anti-politique du néo-humanisme n'avaient pu favoriser l'émergence d'une idée, d'une conception de l'État, dont les romantiques et leurs successeurs avaient cru pouvoir se passer en mettant le Volk, le peuple, à sa place. Dans les combats pour l'unité allemande, les idéaux politiques de la Grèce classique, entachée de Kleinstaaterei, cédèrent le pas devant la force militaire du conquérant et ne s'accommodèrent que difficilement de la monarchie. À l'époque où Plessner rédigea son livre Die verspätete Nation, après la défaite de 1918 et après l'échec de la République de Weimar, un nouveau Reich, le troisième, exploitait et exacerbait les tentatives pour ancrer l'idée de l'État allemand dans l'histoire lointaine et ne trouvait comme fondement de sa fierté, commente Plessner, que primitivité naturelle (naturhafte Ursprünglichkeit) et barbarie éternelle (ewiges Barbarentum) ${ }^{85}$.

Au XIXe siècle, l'Antiquité n'avait donc pu constituer pour le mouvement national et les universitaires qui s'y étaient engagés, ni le véritable point de mire, ni un simple réservoir d'arguments rhétoriques ou idéologiques. La nature et l'importance des références à l'Antiquité avaient fluctué dans un jeu d'influences réciproques entre l'évolution de la question nationale et le développement d'une science de l'Antiquité.

85 H. PLESSNER, Die verspätete Nation. Über die politische Verführbarkeit bürgerlichen Geistes, Francfort, 1974, p. 64 (1 $\mathrm{e}^{\mathrm{e}}$ éd. Zurich, 1935, sous le titre Das Schicksal des deutschen Geistes im Ausgang seiner bürgerlichen Epoche; changement de titre lors de la $2^{\mathrm{e}}$ éd. Stuttgart, 1959). 
En même temps se modifiait la disposition des élites allemandes à l'égard de l'éducation classique orientée sur une Grèce idéalisée et envers la conception prussienne de l'État, qui cédait de plus en plus de terrain face à l'idée séduisante d'un «État de puissance national» (nationaler Machtstaat) ${ }^{86}$.

Hinnerk BRUHNS

CNRS-EHESS / Centre de recherches historiques

54, Bd Raspail

F-75006 Paris

bruhns@msh-paris.fr

86 Voir à ce sujet W.J. MOMMSEN, "Preußisches Staatsbewußtsein und deutsche Reichsidee: Preußen und das Deutsche Reich in der jüngeren deutschen Geschichte", in W.J. MOMMSEN, Der autoritäre Nationalstaat. Verfassung, Gesellschaft und Kultur im deutschen Kaiserreich, Francfort, 1990, p. 66-85. 